\title{
TITLE:
}

\section{Total Synthesis of (-)- Caprazamycin A}

AUTHOR(S):

Nakamura, Hugh; Tsukano, Chihiro; Yasui, Motohiro; Yokouchi, Shinsuke; Igarashi, Masayuki; Takemoto, Yoshiji

\section{CITATION:}

Nakamura, Hugh ...[et al]. Total Synthesis of (-)-Caprazamycin A. Angewandte Chemie 2015, 127(10): 3179-3182

\section{ISSUE DATE:}

2015-03-02

URL:

http://hdl.handle.net/2433/235941

\section{RIGHT:}

This is the peer reviewed version of the following article: Hugh Nakamura, Chihiro Tsukano, Motohiro Yasui, Shinsuke Yokouchi, Masayuki Igarashi, Yoshiii Takemoto, Total Synthesis of (-)-Caprazamycin A, Angewandte Chemie, 127, 10, (3179-3182), (2015) which has been published in final form at https://doi.org/10.1002/ange.201411954. This article may be used for non-commercial purposes in accordance with Wiley Terms and Conditions for Use of Self-Archived Versions.: この論文は出版社版でありません。引用の際には出版社版をご確認じ利用ください。; This is not the published version. Please cite only the published version. 


\title{
Total Synthesis of (-)-Caprazamycin A**
}

\author{
Hugh Nakamura, Chihiro Tsukano, Motohiro Yasui, Shinsuke Yokouchi, Masayuki Igarashi and Yoshiji \\ Takemoto*
}

\begin{abstract}
Caprazamycin A has significant antibacterial activity against Mycobacterium tuberculosis (TB). The first total synthesis is herein reported featuring (i) the scalable preparation of the syn- $\beta$-hydroxy amino acid with a thiourea-catalyzed diastereoselective aldol reaction, (ii) construction of a diazepanone with an unstable fatty acid side chain, and (iii) global deprotection with hydrogenation. This report provides a route for the synthesis of related liponucleoside antibiotics with fatty acid side chains.
\end{abstract}

Caprazamycin A (1) was isolated from Streptomyces sp. MK730 62F2 and is a liponucleoside characterized by a seven-membered diazepanone core with an amino ribose, a uridine and a fatty acid side chain (Figure 1). ${ }^{1}$ Several analogs isolated by Igarashi et al. in 2003 share these features. Caprazamycins have antibacterial activity against Mycobacterium tuberculosis (TB), including multidrug-resistant TB (MDR-TB). Biological studies showed that it is an inhibitor of the peptidoglycan biosynthetic enzyme MraY. ${ }^{2} \mathrm{MraY}$ is essential for bacterial cell growth and is biosynthetically located upstream of an enzyme targeted by $\beta$-lactam and glycopeptide antibiotics (e.g. vancomycin). New antimicrobial agents targeting MraY are expected to be active against vancomycin- and methicillin-resistant Staphylococcus aureus (VRSA and MRSA). ${ }^{3}$ Recently, CPZEN-45, which exhibits more potent activity against $\mathrm{TB}$-including extensively multidrug-resistant TB (XDR-TB), has been developed based on caprazamycins. ${ }^{2 b, 4}$

The complex structure and significant biological activities of caprazamycins have drawn much attention from synthetic chemists. ${ }^{5,6}$ Matsuda and Ichikawa accomplished the first total synthesis of palmitoyl caprazol and caprazol (2), which does not possess a fatty acid side chain. ${ }^{7}$ Shibasaki and Watanabe recently reported the synthesis of $\mathbf{2}$ and the fatty acid side chain. ${ }^{8}$ However, a total synthesis of the caprazamycins has not yet been reported, because of the difficulty in introducing an unstable fatty acid side chain. This has also hampered the total synthesis of related liponucleoside antibiotics, such as liposidomycin $\mathrm{C}(\mathbf{3}){ }^{9}$ Therefore, we initiated a caprazamycin A (1) synthetic

$\left[{ }^{\star}\right] \quad$ H. Nakamura, Dr. C. Tsukano, M. Yasui, S. Yokouchi, Prof. Dr. Y. Takemoto

Graduate School of Pharmaceutical Sciences, Kyoto University Yoshida, Sakyo-ku, Kyoto 606-8501, Japan

E-mail: takemoto@pharm.kyoto-u.ac.jp

Dr. M. Igarashi

Institute of Microbial Chemistry (BIKAKEN)

Kamiosaki, Shinagawa-ku, Tokyo 141-0021 (Japan)

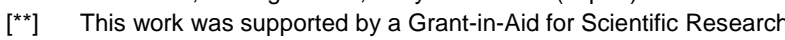
(B) (JSPS KAKENHI no. 23390004, Y.T.), a Grant-in-Aid for JSPS fellows (H.N.) and Meiji Seika Pharma Award in Synthetic Organic Chemistry, Japan (C.T.).

Supporting information for this article is given via a link at the end of the document. project, which would also be applicable to related natural products.
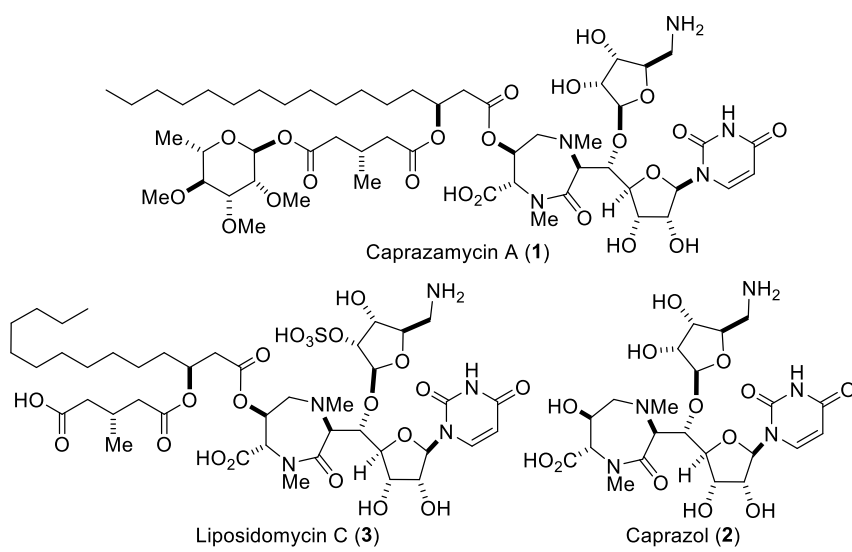

Figure 1. Caprazamycin A (1), caprazol (2) and liposidomycin C (3).

It is challenging to introduce the side chain containing unstable structures. To access caprazamycin A (1), it was envisioned that unstable side chains $\mathbf{4}$ and $\mathbf{5}$ could be introduced to protected caprazol $\mathbf{6}$ as the final step. This would be followed by global deprotection without adversely affecting any functional groups (Scheme 1). Benzyl (Bn), carboxybenzyl (Cbz) and benzyloxymethyl (BOM) protecting groups were selected and are readily removed by Pd-catalyzed hydrogenation. Protected 6 was prepared using (i) the Mitsunobu reaction to construct the seven-membered diazepanone, and (ii) a diastereoselective aldol reaction of isocyanate $\mathbf{8}$ and aldehyde $\mathbf{9}$ with thiourea catalyst $\mathbf{1 0}$ to obtain syn- $\beta$-hydroxy amino acid derivative $7 .{ }^{10}$
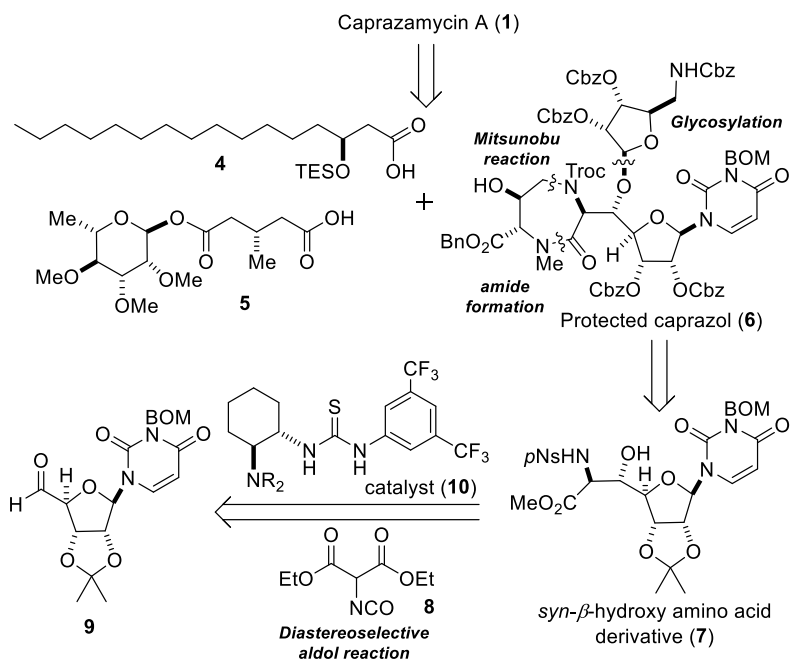

Scheme 1. Retrosynthesis of caprazamycin A (1).

Fatty acid side chains $\mathbf{4}$ and $\mathbf{5}$ were first prepared. $\beta$-Siloxy carboxylic acid $\mathbf{4}$ was synthesized from acid chloride 11 via the modified Noyori asymmetric reduction ${ }^{11}$ 
of $\beta$-ketoester $\mathbf{1 2}^{12}$ (Scheme 2). Enantioselective desymmetrization of 3-methyl glutaric anhydride 13 using cinchona alkaloid catalyst $\mathbf{1 4}$ with Song's procedure ${ }^{13}$ gave carboxylic acid 15 with high enantioselectivity (92\% ee). Condensation of $\mathbf{1 5}$ with L-rhamnose derivative $\mathbf{1 6},{ }^{14}$ followed by removal of the benzyl group of ester 17 with hydrogenolysis gave carboxylic acid $\mathbf{5}$.

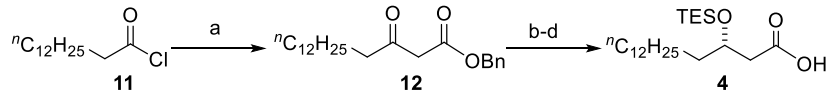
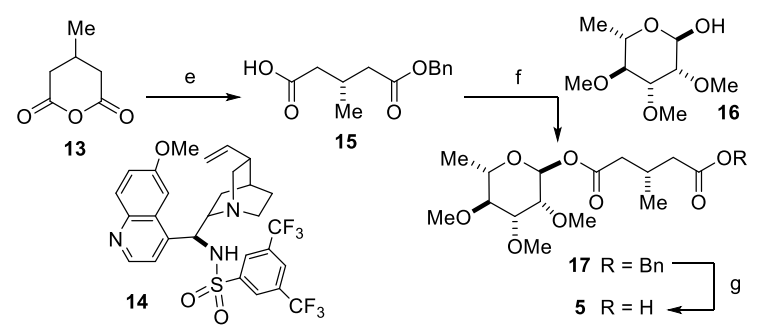

Scheme 2. Synthesis of fatty acid side chains 5 and 6. Reagents and conditions: a) BnOAc, LDA, THF, $-78{ }^{\circ} \mathrm{C}, 51 \%$; b) $\mathrm{H}_{2}$, (S)-BINAP- $\mathrm{RuBr}_{2}(4.0 \mathrm{~mol} \%), \mathrm{MeOH}$ $50{ }^{\circ} \mathrm{C}, 48 \%$, 94\%ee for two steps; c) TESOTf, 2,6-lutidine, $\mathrm{CH}_{2} \mathrm{Cl}_{2}, 0{ }^{\circ} \mathrm{C}, 94 \%$; d) $\mathrm{H}_{2}$, $10 \% \mathrm{Pd} / \mathrm{C}$, EtOAc, $25{ }^{\circ} \mathrm{C}, 92 \%$; e) $\mathrm{BnOH}$, catalyst 14 (10 mol\%), CPME, 86\%, $92 \%$ ee.; f) Ghosez reagent, $\mathrm{CH}_{2} \mathrm{Cl}_{2}, 0{ }^{\circ} \mathrm{C}$, then ${ }^{n} \mathrm{BuLi}$, THF, $48 \%$; g) $\mathrm{H}_{2}, 10 \% \mathrm{Pd} / \mathrm{C}$, EtOAc, $25{ }^{\circ} \mathrm{C}, 92 \% . \mathrm{LDA}=$ lithium diisopropylamide, $\mathrm{CPME}=$ cyclopentyl methyl ether, Ghosez reagent $=1$-chloro- $N, N, 2$ - trimethylpropenylamine.

Construction of the syn- $\beta$-hydroxy amino acid moiety with $S$ configuration at C5' was then investigated. Several strategies have been employed for this in the past, ${ }^{5 \mathrm{~d}, \mathrm{e}, 6 \mathrm{~g}, \mathrm{i}-\mathrm{k}, 7 \mathrm{a}, 8 \mathrm{a}}$ two of which were for the total synthesis of caprazol. One is Sharpless' asymmetric aminohydroxylation of the $\alpha, \beta$-unsaturated ester ${ }^{7 \mathrm{a}}$ and the other is the diastereoselective isocyanoacetate aldol reaction. ${ }^{8 \mathrm{a}} \mathrm{We}$ anticipated that the stereochemistry at C5' could be controlled with a novel diastereoselective aldol reaction using isocyanate $\mathbf{8}$ in the presence of an organocatalyst. ${ }^{10}$

Initially, aldehyde $\mathbf{9}^{15}$ was treated with $\mathbf{8}$ and $\mathrm{Et}_{3} \mathrm{~N}$ (10 mol\%) in toluene to give a mixture of aldol adducts 18a and 18b in $50 \%$ yield with poor diastereoselectivity $(1: 1.8)$ (Table 1, Entry 1). In contrast, treatment with $(S, S)$-thiourea catalyst $\mathbf{1 0 a}(10 \mathrm{~mol} \%)$ in toluene gave desired aldol adduct 18a as the major product in $64 \%$ yield (3.1:1), along with a small amount of byproduct $\mathbf{1 9}$ (Entry 2). The selectivity was improved to $6.5: 1$ by changing to $(S, S)$-thiourea catalyst $\mathbf{1 0 b}$ (10 mol\%) (Entry 3). Formation of byproduct 19 was suppressed by reducing the amount of catalyst $(7 \mathrm{~mol} \%)$ (Entry 4). Use of $(R, R)$-thiourea catalyst 10b $(10 \mathrm{~mol} \%)$ gave undesired diastereomer $\mathbf{1 8 b}$ in $80 \%$ yield with high selectivity (>20:1) (Entry 5). This protocol was also applied to the large scale synthesis of aldol adduct 18a.

Aldol adduct 18a was converted to syn- $\beta$-hydroxy amino acid derivative 7 in good yield by regioselective decarboxylation and transesterification of the resultant thermodynamically stable trans-oxazolidinone in the presence of zinc cluster $\mathrm{Zn}_{4}\left(\mathrm{OCOCF}_{3}\right)_{6} \mathrm{O}$ (Scheme 3). ${ }^{16}$ The minor isomer was removed during these transformations. Following Matsuda and Ichikawa's procedure, ${ }^{7}$ fluoride 21 underwent $\beta$-selective glycosylation, reduction of the azido group, $\mathrm{Cbz}$ protection and hydrolysis under basic conditions to give 22. Carboxylic acid 22 was treated with Ghosez reagent ${ }^{17}$ and coupled with anti- $\beta$-hydroxy amino acid derivative 23. ${ }^{18}$ The TBS group was selectively removed
Table 1. Optimization of diastereoselective aldol reaction.

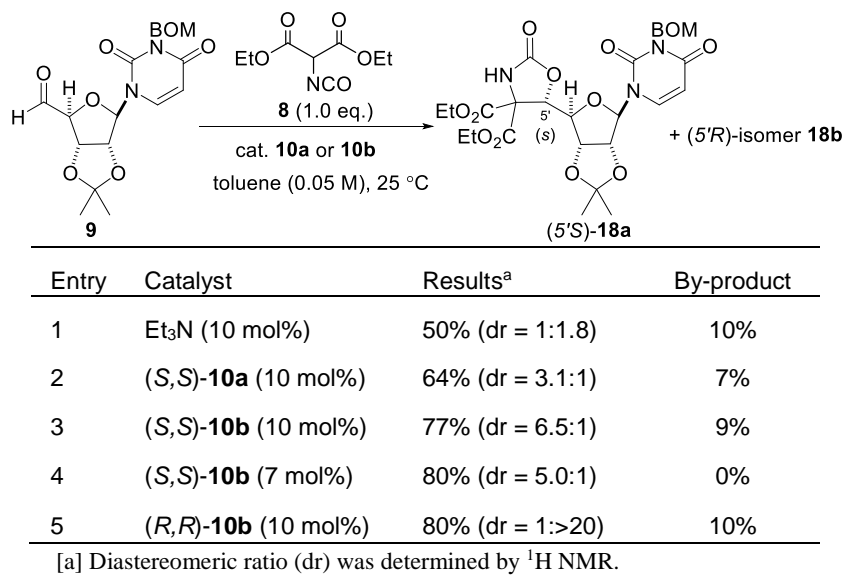

$$
\text { (S,S)-10b }\left(\mathrm{R}={ }^{n} \mathrm{C}_{5} \mathrm{H}_{11}\right) \text { byproduct } 19
$$

and construction of the diazepanone core was extensively investigated. The Mitsunobu reaction of 25 using $\mathrm{PPh}_{3}$ and di-tert-butyl azodicarboxylate (DBAD) proceeded to give the seven-membered ring without epimerization or other side reactions. Finally, protecting group manipulation of $\mathbf{2 6}$ gave protected caprazol $\mathbf{6}$ and the structure was confirmed through conversion to caprazol (2). ${ }^{1,7 a, 8 a}$

With side chain fragments $\mathbf{4}$ and $\mathbf{5}$ and protected caprazol $\mathbf{6}$ in hand, we focused on the introduction of the fatty acid side chain. This side chain readily decomposes through $\beta$-elimination of the $\beta$-acyloxy carbonyl under basic conditions and cleavage of the $O$-acylglycoside under acidic conditions. In fact, attempts to introduce the fatty acid side chain $27^{19}$ to model diazepanone 28 using EDCI caused $\beta$-elimination to give unsaturated carboxylic acid $\mathbf{3 0}$ instead of desired 29 (Scheme 4). DCC and PyBOP were also ineffective. The $\beta$-hydroxy ester of diazepanone may also decompose through $\beta$-elimination and a retro-aldol reaction. Thus, fragments $\mathbf{4}$ and $\mathbf{5}$ were introduced in a stepwise manner.

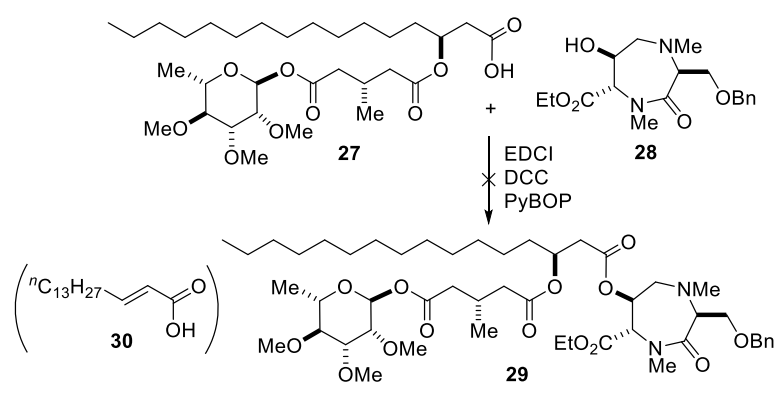

Scheme 4. Initial attempts to introduce fatty acid side chain (27).

The final stage of this synthesis began with coupling $\beta$-siloxy carboxylic acid 4 with protected caprazol 6 without epimerization (Scheme 3). The Troc group was removed under mild conditions without touching the unstable $\beta$-acyloxy moiety. This was followed by reductive amination. After removal of the TES group, carboxylic acid 5 was introduced to resultant alcohol $\mathbf{3 1}$ using Yamaguchi 
conditions to give protected caprazamycin A (32). ${ }^{8 b}$ Finally, global deprotection with hydrogenation in the presence of Pd black was successful without side-chain decomposition. This completed the first total synthesis of caprazamycin A (1). The ${ }^{1} \mathrm{H}$ and ${ }^{13} \mathrm{C}$ NMR, IR and HRMS for this matched those of the natural product. ${ }^{20}$

In summary, we have accomplished the first total synthesis of caprazamycin A in 23 steps (longest linear sequence from aldehyde 9). The key points are (i) scalable synthesis of the syn- $\beta$-hydroxy amino acid moiety with a thiourea-catalyzed diastereoselective aldol reaction, (ii) maintaining the structural integrity of the diazepanone core during introduction of the fatty acid side chain, and (iii) global deprotection with hydrogenation. This is the first report detailing the introduction of the unstable fatty acid side chain. This should allow the synthesis of related liponucleoside antibiotics.
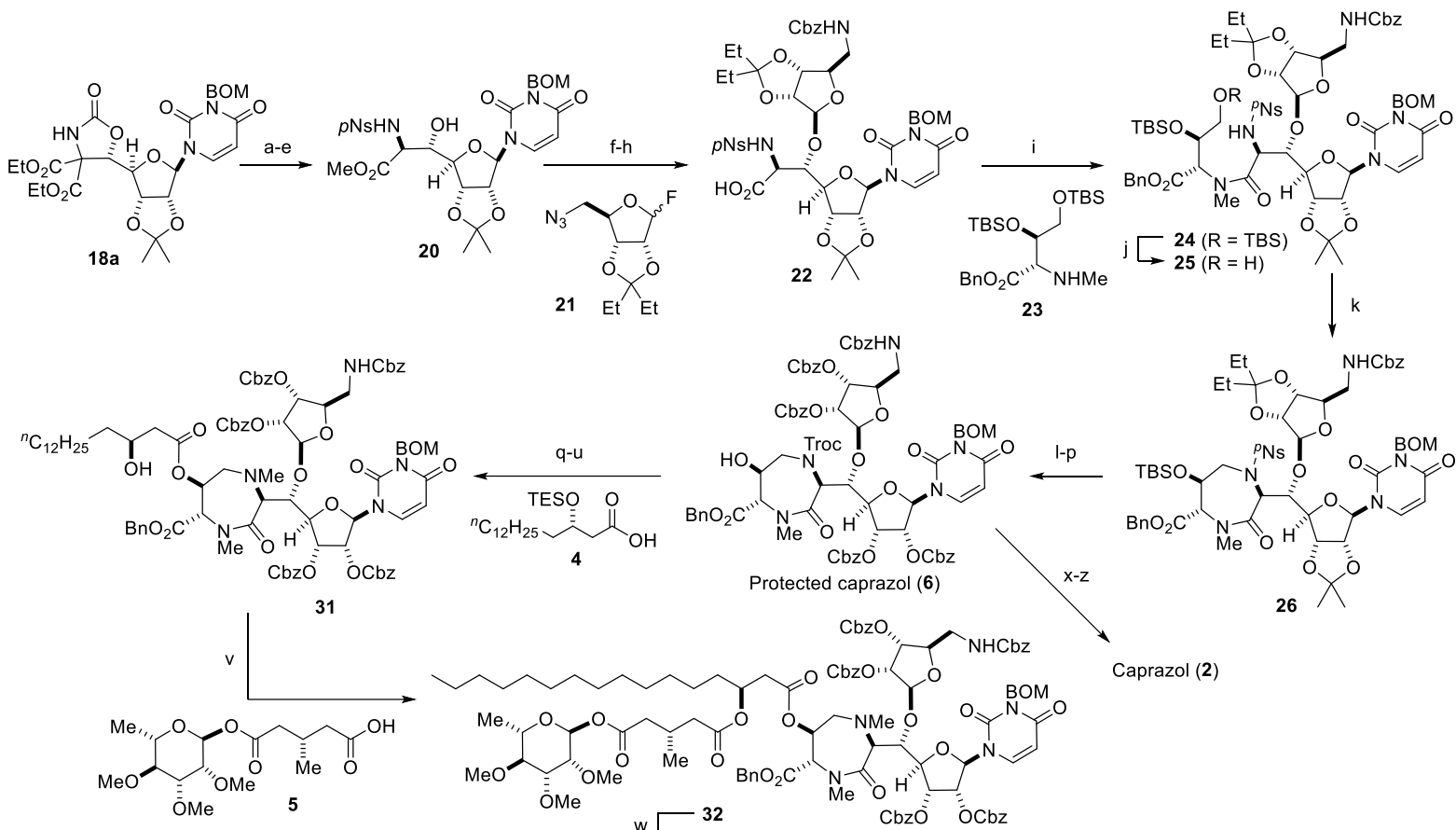

Caprazol (2)

Scheme 3. Total synthesis of caprazamycin A (1). Reagents and conditions: a) aq. $\mathrm{KOH}$, THF, 0 to $25^{\circ} \mathrm{C}$; b) $\mathrm{DBU}, \mathrm{THF}, 70{ }^{\circ} \mathrm{C}, 86 \%(2 \mathrm{steps})$; c) $\mathrm{Zn}_{4}(\mathrm{OCOCF} 3)_{6} \mathrm{O}(3.2 \mathrm{~mol} \%)$, aq $\mathrm{NaHCO}_{3}, 0$ to $25^{\circ} \mathrm{C}$; h) $\mathrm{Ba}(\mathrm{OH})_{2} \cdot 8 \mathrm{H}_{2} \mathrm{O}, \mathrm{THF} / \mathrm{H}_{2} \mathrm{O}=4: 1,0$ to $25{ }^{\circ} \mathrm{C}$; i) Ghosez reagent, $\mathrm{CH}_{2} \mathrm{Cl}_{2}, 0{ }^{\circ} \mathrm{C}$ then 23 , aq. $\mathrm{NaHCO}_{3}, 0{ }^{\circ} \mathrm{C}, 46 \%(3$ steps $)$; j) $\mathrm{CSA}, \mathrm{MeOH}^{\circ} \mathrm{CH}_{2} \mathrm{Cl}_{2}=1: 1$, $0{ }^{\circ} \mathrm{C}, 68 \%$ (17\% for recovered 24, b.r.s.m. $82 \%$ ); k) $\mathrm{PPh}_{3}$, DBAD, toluene, $0{ }^{\circ} \mathrm{C}, 75 \%$; l) $\mathrm{K}_{2} \mathrm{CO}_{3}, \mathrm{PhSH} \mathrm{MeCN}, 0$ to $25{ }^{\circ} \mathrm{C}, 73 \%$; m) TrocCl, DMAP, pyridine, $\mathrm{CH}_{2} \mathrm{Cl} 2,0$ to $25{ }^{\circ} \mathrm{C}$, $79 \%$; n) TsOH$\cdot \mathrm{H}_{2} \mathrm{O}, \mathrm{MeOH}, 60{ }^{\circ} \mathrm{C}, 41 \%$ and diol having penthylidene acetal $(21 \%)$; o) $\mathrm{CbzCl}, \mathrm{DMAP}, \mathrm{CH}_{2} \mathrm{Cl}_{2}, 0$ to $\left.25{ }^{\circ} \mathrm{C} ; \mathrm{p}\right) \mathrm{pTsOH} \cdot \mathrm{H}_{2} \mathrm{O}, \mathrm{MeOH}, 25$ to $60{ }^{\circ} \mathrm{C}, 71 \%(2$ steps); q) 4, EDCI, DMAP, $\mathrm{CH}_{2} \mathrm{Cl}_{2}, 0$ to $25{ }^{\circ} \mathrm{C}$; r) $\mathrm{Zn}$, AcOH/THF, $25{ }^{\circ} \mathrm{C}$; s) $\mathrm{AcOH}, \mathrm{ClCH}_{2} \mathrm{CH}_{2} \mathrm{Cl}, 25{ }^{\circ} \mathrm{C}$; t $)\left(\mathrm{CH}_{2} \mathrm{O}\right)_{\mathrm{n}}, \mathrm{NaBH}(\mathrm{OAc})_{3}, \mathrm{AcOH} / \mathrm{ClCH} \mathrm{CH}_{2} \mathrm{Cl}, 25{ }^{\circ} \mathrm{C}$; u) $\mathrm{HF}$.py, THF, $0{ }^{\circ} \mathrm{C}$ to $25{ }^{\circ} \mathrm{C}, 43 \%$ (5 steps); v) 5, 2,4,6- trichlorobenzoyl chloride, DMAP, Et $3 \mathrm{~N}, 0$ to $25{ }^{\circ} \mathrm{C}, 64 \%$; w) Pd black, EtOH/HCO $\mathrm{HC}_{2} \mathrm{H}=20: 1,25{ }^{\circ} \mathrm{C}, 98 \%$; $\left.\mathrm{x}\right) \mathrm{Zn}, \mathrm{AcOH} / \mathrm{THF}, 25$ to $50{ }^{\circ} \mathrm{C}$; y) $\left(\mathrm{CH}_{2} \mathrm{O}\right)_{n}, \mathrm{NaBH}(\mathrm{OAc})_{3}, \mathrm{AcOH} / \mathrm{CH}_{2} \mathrm{Cl}_{2}, 25{ }^{\circ} \mathrm{C}$, quant. (2 steps); z) Pd black, EtOH/ $\mathrm{HCO}_{2} \mathrm{H}=10: 1,25{ }^{\circ} \mathrm{C}, 46 \%$. DBU = 1,8-diazabicyclo[5.4.0]undec-7-ene, $p \mathrm{Ns}=$ 4-nitrobenzenesulfonyl, Ghosez reagent $=1$-chloro- $N, N, 2$-trimethylpropenylamine, $\mathrm{CSA}=10$-camphor sulfonic acid, $\mathrm{DBAD}=$ di-tert-butyl azodicarboxylate, TrocCl $=$ 2,2,2-trichloroethyl chloroformate, DMAP $=\mathrm{N}, \mathrm{N}$-dimethyl-4-aminopyridine, Cbz $=$ benzyloxycarbonyl, $p$ Ts $=p$-toluenesulfonyl, EDCI $=1$-Ethyl-3- $(3$-dimethylaminopropyl $)$ carbodiimide

Keywords: antibiotics - natural products - total synthesis • caprazamycin • liponucleoside

[1] a) M. Igarashi, N. Nakagawa, N. Doi, S. Hattori, H. Naganawa, M. Hamada, J. Antibiot. 2003, 56, 580; b) T. Takeuchi, M. Igarashi, H. Naganawa, JP P2003-12687A, 2003; c) M. Igarashi, Y. Takahashi, T. Shitara, H. Nakamura, H. Naganawa, T. Miyake, Y. Akamatsu, J. Antibiot. 2005, 58, 327; d) C. Dini, Curr. Top. Med. Chem. 2005, 5, 1221; e) T. D. H. Bugg, A. J. Lloyd, D. I. Roper, Infect. Disord. Drug Targets, 2006, 6, 85 .

[2] a) C. Dini, P. Collette, N. Drochon, J. C. Guillot, G. Lemoine, P. Mauvais, J. Aszodi, Bioorg. Med. Chem. Lett. 2000, 10, 1839; b) Y. Ishizaki, C. Hayashi, K. Inoue, M. Igarashi, Y. Takahashi, V. Pujari, D. C. Crick, P. J. Brennan, A. Nomoto, J. Biol. Chem. 2013, 288, 30309.

[3] K. Kimura, T. D. H. Bugg, Nat. Prod. Rep. 2003, 20, 252.

[4] a) Y. Takahashi, M. Igarashi, T. Miyake, H. Soutome, K. Ishikawa, Y. Komatsuki, Y. Koyama, N. Nakagawa, S. Hattori, K. Inoue, N. Doi, Y. Akamatsu, J. Antibiot. 2013, 66, 171; b) S. N. M. Hanif, A. J. Hickey, L. Garcia-Contreras, J. Pharm. Biomed. Anal. 2014, 88, 370.
[5] Synthetic studies toward caprazamycins: a) S. Hirano, S. Ichikawa, A Matsuda, Bioorg. Med. Chem. 2008, 16, 5123; b) S. Ichikawa, Chem Pharm. Bull. 2008, 56, 1059; c) K. Ii, S. Ichikawa, B. Al-Dabbagh, A. Bouhss, A. Matsuda, J. Med. Chem. 2010, 53, 3793; d) A. P. Spork, S Koppermann, B. Dittrich, R. Herbst-Irmer, C. Ducho, Tetrahedron: Asymmetry 2010, 21, 763; e) F. Sarabia, C. Vivar-García, C. García-Ruiz, L. Martín-Ortiz, A. Romero-Carrasco, J. Org. Chem. 2012, 77, 1328; f) C. Tsukano, S. Yokouchi, A. L. Girard, T. Kuribayashi, S Sakamoto, T. Enomoto, Y. Takemoto, Org. Biomol. Chem. 2012, 10, 6074; g) H. Miyaoka, J. Wada, E. Kawashima, Heterocycles 2014, 88, 719 .

[6] Synthetic studies towards related liponucleoside antibiotics: a) M. R. Spada, M. Ubukata, K. Isono, Heterocycles 1992, 34, 1147; b) S. Knapp, S. Nandan, L. Resnick, Tetrahedron Lett. 1992, 33, 5485; c) K. S. Kim, I. H. Cho, Y. H. Ahn, J. I. Park, J. Chem. Soc., Perkin Trans 1 1995, 1783; d) W. J. Moore, F. A. Luzzio, Tetrahedron Lett. 1995, 36, 6599; e) K. S. Kim, C. S. Cheong, J. S. Hahn, J. I. Park, Bull. Korean Chem. Soc. 1997, 18, 465; f) Y. L. Merrer, C. Gravier-Pelletier, M. Gerrouache, J. C. Depezay, Tetrahedron Lett. 1998, 39, 385; g) K. S. Kim, Y. H. Ahn, Tetrahedron: Asymmetry 1998, 9, 3601; h) S. Knapp, G. J. Morriello, S. R. Nandan, T. J. Emge, G. A. Doss, R. T. Mosley, L. Chen, J. Org. Chem. 2001, 66, 5822; i) C. Gravier- Pelletier, M. Milla, Y. L. 
Merrer, J. C. Depezay, Eur. J. Org. Chem. 2001, 16, 3089; j) S. Knapp, G. J. Morriello, G. A. Doss, Org. Lett. 2002, 4, 603; k) B. Drouillat, O Poupardin, Y. Bourdreux, C. Greck, Tetrahedron Lett. 2003, 31, 2781; 1) A. Yamashita, E. B. Norton, R. T. Williamson, D. M. Ho, S. Sinishtaj, T. S. Mansour, Org. Lett. 2003, 5, 3305; m) F. Sarabia, L. Martin-Ortiz, F. J. Lopez-Herrera, Org. Lett. 2003, 5, 3927; n) N. Nakajima, T. Isobe, S. Irisa, M. Ubukata, Heterocycles 2003, 59, 107; o) S. Fukunishi, M. Ubukata, N. Nakajima, Heterocycles 2005, 66, 129; p) Y. Bourdreux, B. Drouillat, C. Greck, Lett. Org. Chem. 2006, 3, 368; q) S. Fukunishi, M. Ubukata, N. Nakajima, Heterocycles 2007, 73, 627; r) O. Monasson, M. Ginisty, G. Bertho, C. Gravier-Pelletier, Y. L. Merrer, Tetrahedron Lett. 2007, 48, 8149; s) X. H. Xu, A. E. Trunkfield, T. D. H. Bugg, F. L. Qing, Org. Biomol. Chem. 2008, 6, 157; t) O. Monasson, M. Ginisty, J. Mravljak, G. Bertho, C. Gravier-Pelletier, Y. L. Merrer, Tetrahedron: Asymmetry 2009, 20, 2320; u) M. J. Fer, P. Doan, T. Prangé, S. Calvet-Vitale, C. Gravier-Pelletier, J. Org. Chem. 2014, 79, 7758; v) A P. Spork, M. Büschleb, O. Ries, D. Wiegmann, S. Boettcher, A. Mihalyi, T. D. H. Bugg, C. Ducho, Chem. Eur. J. 2014, 20, 15292.

[7] a) S. Hirano, S. Ichikawa, A. Matsuda, Angew. Chem. Int. Ed. 2005, 44 , 1854; b) S. Hirano, S. Ichikawa, A. Matsuda, J. Org. Chem. 2007, 72, 9936; c) S. Hirano, S. Ichikawa, A. Matsuda, J. Org. Chem. 2008, 73, 569.

[8] a) P. Gopinath, L. Wang, H. Abe, G. Ravi, T. Masuda, T. Watanabe, M. Shibasaki, Org. Lett. 2014, 16, 3364; b) P. Gopinath, T. Watanabe, M. Shibasaki, J. Org. Chem. 2012, 77, 9260.

[9] a) K. Isono, M. Uramoto, H. Kusakabe, K. Kimura, K. Izaki, C. C. Nelson, J. A. McCloskey, J. Antibiot. 1985, 38, 1617; b) K. Kimura, Y Ikeda, S. Kagami, M. Yoshihama, K. Suzuki, H. Osada, K. Isono, $J$. Antibiot. 1998, 51, 1099; c) M. Ubukata, K. Kimura, K. Isono, C. C. Nelson, J. M. Gregson, J. A. McCloskey, J. Org. Chem. 1992, 57, 6392; d) K. Kimura, Y. Ikeda, S. Kagami, M. Yoshihama, M. Ubukata, Y. Esumi, H. Osada, K. Isono, J. Antibiot. 1998, 51, 647; e) K. Kimura, S.
Kagami, Y. Ikeda, H. Takahashi, M. Yoshihama, H. Kusakabe, H. Osada, K. Isono, J. Antibiot. 1998, 51, 640.

[10] S. Sakamoto, N. Kazumi, Y. Kobayashi, C. Tsukano, Y. Takemoto, Org. Lett. 2014, 16, 4758

[11] a) R. Noyori, H. Takaya, Acc. Chem. Res. 1990, 23, 345; b) V. Ratovelomanana-Vidal, C. Girard, R. Touati, J. P. Tranchier, B. Ben Hassine, J. P. Genêt, Adv. Synth. Catal. 2003, 345, 261.

[12] D. F. Taber, P. B. Deker, H. M. Fales, T. H. Jones, H. A. Lloyd, J. Org. Chem. 1988, 53, 2968.

[13] a) S. H. Oh, H. S. Rho, J. W. Lee, J. E. Lee, S. H. Youk, J. Chin, C. E. Song, Angew. Chem. Int. Ed. 2008, 47, 7872; b) T. Honjo, T. Tsumura, S. Sano, Y. Nagao, K. Yamaguchi, Y. Sei, Synlett, 2009, 20, 3279.

[14] a) M. S. Arias-Pérez, M. S. López, M. J. Santos, J. Chem. Soc., Perkin Trans. 2, 2002, 1549; b) D. A. Evans, W. C. Black, J. Am. Chem. Soc. 1993, 115,4497

[15] Aldehyde 9 was prepared from commercially available uridine in three steps. See Supporting Information.

[16] T. Ohshima, T. Iwasaki, Y. Maegawa, A. Yoshiyama, K. Mashima, $J$ Am. Chem. Soc. 2008, 130, 2944

[17] A. Devos, J. Remion, A.-M. Frisque-Hesbain, A. Colens, L. Ghosez, $J$. Chem. Soc., Chem. Commun. 1979, 1180.

[18] Amine 23 was synthesized from L-(+)-diethyl tartrate in ten steps. See Supporting Information.

[19] Carboxylic acid 27 was prepared from the precursor of $\mathbf{4}$ and $\mathbf{5}$ as described by Shibasaki and Watanabe et al (ref 8b).

[20] The NMR spectrum of caprazamycin A was dependent on concentration and pKa. Thus, the NMR was measured in DMSO- $\mathrm{d}_{6} / \mathrm{D}_{2} \mathrm{O} / \mathrm{DCO}_{2} \mathrm{D}$ (20:1:1 for comparison. Also see Supporting Information. 